\title{
Barani Mash a Newly Developed Disease Resistant and High Yielding Mash Cultivar for Rainfed Areas of Punjab, Pakistan
}

\author{
Ghulam Rabbani $^{1}$, Uzma Javed ${ }^{1 *}$, Javed Iqbal ${ }^{2}$, Ruqeah Mustafa ${ }^{3}$, Ghulam Shabbir ${ }^{2}$ and Fida Hassan \\ $\mathrm{Shah}^{4}$
}

${ }^{1}$ Pulses Section, Barani Agricultural Research Institute Chakwal, Pakistan; ${ }^{2}$ Plant Breeding and Genetics Dept., PMAS Arid Agriculture University Rawalpindi, Pakistan; ${ }^{3}$ Oilseeds Section, Barani Agricultural Research Institute Chakwal, Pakistan; ${ }^{4}$ Groundnut Section, Barani Agricultural Research Institute Chakwal, Pakistan.

\begin{abstract}
New cultivars development is a continuous process as older genotypes become increasingly susceptible to biotic and abiotic stresses. The newly developed mash cultivar "Barani Mash" is an elite mash cultivar with yield potential of $1743 \mathrm{~kg} / \mathrm{ha}$. This cultivar has resistance against diseases and lodging, the pod size is medium and 100-seed weight is $4.73 \mathrm{~g}$. It has been developed through screening from the local material. It was further evaluated in yield trials for six years from 2012 to 2016 . The line 11CM-707 had higher yield than check cultivars per all replicated yield trials. In addition, it has resistance to yellow mosaic. The main yield contributing characters were number of pods per plant, number of primary branches per plant, yield per plant and 100-kernal weight. Based on desirable traits and higher yield, this line was approved with name of "Barani Mash" by the Punjab Seed Council for its cultivation on commercial level in rainfed areas of Punjab. "Barani Mash" will prove to be a good alternative of existing cultivars and cultivation of this cultivar would help to increase the productivity and income of mash growers in Barani tract.

Received | August 11, 2020; Accepted | October 25, 2020; Published | February 06, 2021

*Correspondence | Uzma Javed, Pulses Section, Barani Agricultural Research Institute Chakwal, Pakistan; Email: uzma_raja18@yahoo.com

Citation | Rabbani, G., U. Javed, J. Iqbal, R. Mustafa, G. Shabbir and F.H. Shah. 2021. Barani mash a newly developed disease resistant and high yielding mash cultivar for rainfed areas of Punjab, Pakistan. Sarbad Journal of Agriculture, 37(1): 115-119.

DOI | http://dx.doi.org/10.17582/journal.sja/2021/37.1.115.119

Keywords | New mash cultivar, Barani Mash, Rainfed areas, High yielding, Disease resistant
\end{abstract}

\section{Introduction}

$\mathrm{B}$ lack gram (Vigna mungo) or Mash belongs to family leguminosae, is an important pulse crop of Pakistan. Southern Asian countries especially India, Myanmar, Thailand and Bangladesh are the main growing regions of the mash crop. Mash is a densely hairy erect or sub-erect annual herb. It has a tap root system that produces branched roots with smooth and rounded nodules. The pods of the crop are cylindrical, narrow and up to $6 \mathrm{~cm}$ long. It contains amino acids, sulphur, cysteine, methionine, and lysine which are excellent components of balanced human diet. It is also a good source of iron, sodium, potassium, calcium, magnesium, phosphorus, sulphur, copper, and vitamins. Mash being an important source of protein is increasingly used as a substitute for animal protein. The mash crop splits or dried whole seeds are used to make curries, sours and dahl, and are added to various fried or spiced dishes. Sprouted seeds of mash are also utilized as a quality vegetable ingredient in different parts of world. Therefore, emphasis should be given to develop Mash cultivars which may ultimately be helpful to achieve self-sufficiency in the production of pulses.

During 2018-19, mash crop was cultivated over an area of $14.1(000)$ hectares in Pakistan, with a total 
production of 6.9(000) tones (GOP, 2018-19). Mash crop is cultivated in all four provinces of Pakistan; however, Punjab has the largest area under mash cultivation.

In Punjab, a total of $12.17(000)$ hectares is under mash cultivation giving a production of 3.6(000) tones. Rain fed areas contribution is $84 \%$ while irrigated contributes only 16\% (AMIS, 2017-18). A wide range of agro-ecological zones of Pakistan are suitable for mash cultivation. This crop has potential to produce high yield, however, due to a variety of factors, crop yield remains below its potential. Therefore, our country is not self-sufficient in mash production, each year government spends a substantial amount of funds and spends its foreign exchange to import mash for consumption. Last year alone, Pakistan imported 64.08 thousand tons of mash with value of 7824.55 million rupees (AMIS, 2019-20).

Mash bean introduction in cereal cropping system can potentiallybenefit byimproving netreturns, soilfertility and agriculture sustainability. Hence, development of a high yielding and disease resistant mash cultivars is of vital importance. Barani Agricultural Research Institute, Chakwal, Pakistan has developed a high yielding disease resistant mash cultivar "Barani Mash" that has the potential to produce $1743 \mathrm{~kg} \mathrm{ha}^{-1}$. This paper illustrates breeding history of cultivar "Barani Mash". This cultivar is which approved by the Punjab Seed Council for general cultivation in rainfed areas of Punjab, Pakistan.

\section{Materials and Methods}

The procedure developed and adopted by Naeemud-Din et al. (2009) was followed for varietal development. An elite line of Mash genotype 11CM707 was screened from local material. Performance evaluation of this line was carried out in different replicated yield trials along with check cultivars for three consecutive years (2012 to 2014) at BARI, Chakwal Pakistan. Regional adaptability, plant protection and agronomic trials were conducted during 2015 and 2016. All the trials were conducted according to randomized complete block design (RCBD). Plant to plant and row to row spacing was kept at 10 and $30 \mathrm{~cm}$, respectively. Standard cultural practices and inputs including fertilizer were used to conduct the trials at different environments. Field trials were conducted on sandy loam soil. Data were recorded for different plant traits in various experiments and analyzed statistically using software statistix 8.1.

\section{Results and Discussion}

\section{Yield performance trials}

Mash preliminary, regular and micro-yield trials were conducted during 2012, 2013 and 2014, respectively at Barani Agricultural Research Institute (BARI) Chakwal, Punjab, Pakistan. National uniform yield trials were performed during 2015 and 2016. Spot examination was performed during 2018 and its case was submitted to the Expert Sub-Committee for approval. All the experts/members of Expert SubCommittee unanimously recommended the case of this new mash line 11 CM-707 "BARANI MASH" developed by Barani Agricultural Research Institute, Chakwal for the consideration by the Punjab Seed Council for its approval. Finally, it was approved by the Punjab Seed Council for general cultivation in the Pothowar area of the Punjab province. Table 2 shows the significant differences $(\mathrm{P}<0.05)$ among the varietal means for seed yield in preliminary yield trial of mash. Genotype 11 CM-707 produced a seed yield of $628 \mathrm{~kg} \mathrm{ha}^{-1}$ and was ranked $4^{\text {th }}$ among the tested genotypes. $11 \mathrm{CM}-707$ produced 24\% higher seed yield than check cultivar Arooj. Likewise, 11 CM707 produced $11 \%$ and $16 \%$ higher seed yield than check cultivars Chakwal Mash and Arooj respectively in the regular yield trials, as mentioned in Table 3. In micro- yield trial on an average the proposed line 11 CM-707 produced 23\% and 25\% higher seed yield than check cultivars Chakwal Mash and Arooj (Table 4).

Table 1: Chronological development of BARANIMASH (11CM-707).

\section{Year Pedigree Remarks}

2012 11CM-707 Preliminary Yield Trial

2013 11CM-707 Regular Yield Trial

2014 11CM-707 Micro Yield Trial

2015 11CM-707 National Uniform Yield Trial (5 locations); Sowing dates Trial; Fertilizer Requirement Trial; Screening against Diseases

2016 11CM-707 National Uniform Yield Trial (5 locations); Sowing dates Trial; Fertilizer Requirement Trial; Screening against Diseases

2018 11CM-707 Spot Examination Report 
Table 2: Seed yield of various mash genotypes in PYT -2012 .

\begin{tabular}{lll} 
S. No & ENTRIES & Yield (kg/ha) \\
\hline 1 & $11 \mathrm{CM}-709$ & 750 \\
2 & $11 \mathrm{CM}-705$ & 681 \\
3 & $11 \mathrm{CM}-710$ & 653 \\
4 & $11 \mathrm{CM}-707$ & 628 \\
5 & $11 \mathrm{CM}-701$ & 618 \\
6 & $11 \mathrm{CM}-706$ & 618 \\
7 & MASH-97 & 614 \\
8 & CH-MASH (c) & 606 \\
9 & $11 \mathrm{CM}-704$ & 542 \\
10 & $11 \mathrm{CM}-703$ & 528 \\
11 & $11 \mathrm{CM}-702$ & 514 \\
12 & Arooj (c) & 500 \\
13 & $11 \mathrm{CM}-708$ & 424 \\
& LSD (0.05) & 67.81 \\
& CV (\%) & 6.79
\end{tabular}

Table 4: Seed yield of various mash genotypes in MYT2014.

$\begin{array}{lll}\text { Sr. No } & \text { Genotypes } & \text { Seed yield (kg/ha) } \\ 1 & 11 \mathrm{CM}-707 & 528 \\ 2 & 11 \mathrm{CM}-705 & 500 \\ 3 & 11 \mathrm{CM}-703 & 486 \\ 4 & 11 \mathrm{CM}-709 & 458 \\ 5 & 11 \mathrm{CM}-701 & 438 \\ 6 & 11 \mathrm{CM}-710 & 438 \\ 7 & \text { CH-MASH(c) } & 431 \\ 8 & \text { Arooj (c) } & 424 \\ 9 & 11 \mathrm{CM}-702 & 382 \\ 10 & 11 \mathrm{CM}-706 & 375 \\ 11 & 11 \mathrm{CM}-704 & 361 \\ \text { LSD(0.05) } & & 126.27 \\ \text { CV \% } & & 09.09\end{array}$

Table 3: Seed yield of various mash genotypes in RYT2013.

\begin{tabular}{lll} 
Sr. No. & Entries & Seed yield $(\mathbf{K g} / \mathbf{h a})$ \\
1 & $11 \mathrm{CM}-707$ & 1743 \\
2 & $11 \mathrm{CM}-705$ & 1667 \\
3 & $11 \mathrm{CM}-709$ & 1653 \\
4 & $11 \mathrm{CM}-701$ & 1604 \\
5 & $11 \mathrm{CM}-703$ & 1587 \\
6 & $11 \mathrm{CM}-706$ & 1580 \\
7 & CH-MASH(c) & 1577 \\
8 & Arooj (c) & 1506 \\
9 & MASH-97(c) & 1306 \\
10 & $11 \mathrm{CM}-710$ & 1194 \\
11 & $11 \mathrm{CM}-702$ & 1160 \\
12 & $11 \mathrm{CM}-704$ & 1083 \\
& LSD $(0.05)$ & 86.69 \\
CV (\%) & & 7.39 \\
\hline
\end{tabular}

Table 5: Yield performance of 11CM-707 $(\mathrm{kg} / \mathrm{ha})$ at different locations in NUYT 2015.

\begin{tabular}{|c|c|c|c|c|c|c|c|c|}
\hline \multirow[t]{2}{*}{ Entry No. } & \multirow[t]{2}{*}{ Entry name } & \multirow[t]{2}{*}{ Source } & \multicolumn{6}{|c|}{ Locations (kg/Ha) } \\
\hline & & & ISD & FSD & FJ & CHK & DIK & Entry mean \\
\hline 1 & 10CM-707 & BARI & 182 & 705 & 313 & 825 & 1306 & 666 \\
\hline 2 & 10CM-703 & BARI & 167 & 855 & 601 & 883 & 819 & 665 \\
\hline 3 & 11CM-707 & BARI & 154 & 640 & 559 & 998 & 657 & 602 \\
\hline 4 & 10CM-702 & BARI & 118 & 717 & 361 & 869 & 755 & 564 \\
\hline 5 & Arooj & Check & 160 & 592 & 438 & 984 & 418 & 518 \\
\hline 6 & NARC-Mash -014 & NARC, ISD & 381 & 480 & 420 & 1013 & 223 & 503 \\
\hline 7 & Mash-010-2 & NARC & 145 & 490 & 389 & 854 & 560 & 488 \\
\hline 8 & Mash-010-1 & NARC & 238 & 586 & 378 & 1013 & 162 & 475 \\
\hline \multicolumn{3}{|c|}{ Location mean } & 193 & 633 & 432 & 930 & 613 & \\
\hline
\end{tabular}

\section{Agronomic trials}

In the agronomic trials conducted during 2015 and 2016 at BARI, Chakwal, on an average of both years, $11 \mathrm{CM}-707$ produced the highest yield of $934.9 \mathrm{~kg}$ $\mathrm{ha}^{-1}$ when planted on $10^{\text {th }}$ July followed by $1^{\text {st }}$ July which produced $868.15 \mathrm{~kg} \mathrm{ha}^{-1}$ (Table 8). Therefore, optimum sowing time for genotype $11 \mathrm{CM}-707$ is from July 1 to 10 .

Regional adaptability trials the National Uniform Yield Trials (NUYT) during 2015 and 2016. Mash genotype 11 CM-707 was found higher yielder as compared to check cultivar Arooj. On an average, genotype 11 CM-707 showed $22.0 \%$ higher seed yield than the check cultivar Arooj based on two-year yield data collected on field trilas on ten locations, (Table 7). 
Table 6: Yield performance of 11CM-707 $(\mathrm{Kg} / \mathrm{ha})$ at different locations in NUYT 2016.

\begin{tabular}{lllllllll} 
Entry No. Entry name & Source & \multicolumn{6}{c}{ Locations (Kg/Ha) } \\
& & & U Kot & FSD & Quetta & ISD & CHK & Entry mean \\
1 & 11CM-707 & BARI & 696 & 499 & 1346 & 1268 & 1368 & 1035 \\
2 & MASH-010-2 & NARC & 506 & 664 & 860 & 1753 & 1271 & 1011 \\
3 & NARC Mash-3 & NARC & 728 & 754 & 929 & 1173 & 1250 & 967 \\
4 & NARC-Mash 2014 & NARC & 715 & 436 & 826 & 1453 & 1389 & 964 \\
5 & 10 CM-707 & BARI & 742 & 567 & 944 & 903 & 1278 & 887 \\
6 & 10CM-702 & BARI & 567 & 439 & 738 & 1329 & 1229 & 860 \\
7 & 10CM-703 & BARI & 671 & 425 & 446 & 1354 & 1313 & 842 \\
8 & Arooj & Check & 894 & 494 & 964 & 863 & 931 & 829 \\
Location Mean & & 690 & 535 & 882 & 1262 & 1253 &
\end{tabular}

Table 7: Average yield performance of 11CM-707at 10 different locations.

\begin{tabular}{llll} 
Year & Locations & \multicolumn{2}{c}{ Seed yield (kg/ha) } \\
& & 11CM-707 & Arooj (c) \\
2015 & 5 & 602 & 518 \\
2016 & 5 & 1035 & 829 \\
Average & 819 & 674 \\
Yield of 11 CM-707 & & $22 \%$
\end{tabular}

Increase/Decrease (\%) over Checks.

Table 8: Seed yield (kg/ha) as influenced by various sowing dates of $11 C M-707$.

\begin{tabular}{llllllll} 
Cultivar & Year & \multicolumn{6}{c}{ Sowing dates (Yield in Kg/ha) } \\
& & $\mathbf{1 0}^{\text {th }}$ & $\mathbf{2 0}^{\text {th }}$ & $\mathbf{1}^{\text {st }}$ July & $\mathbf{1 0}^{\text {th }}$ & $\mathbf{2 0}^{\text {th }}$ & $\mathbf{1}^{\text {st }} \mathbf{A u}-$ \\
& & June & June & & July & July & gust \\
$11 \mathrm{CM}-707$ & 2015 & 800.0 & 820.2 & 890.5 & 990.5 & 800.0 & 760.5 \\
$11 \mathrm{CM}-707$ & 2016 & 838.8 & 846.2 & 845.8 & 879.3 & 852.6 & 732.6 \\
Means & 819.4 & 833.2 & 868.15 & 934.9 & 826.3 & 746.55
\end{tabular}

Table 9: Seed yield as affected by different levels of fertilizers.

\begin{tabular}{|c|c|c|c|c|c|c|}
\hline \multirow{2}{*}{$\begin{array}{l}\text { Sr. } \\
\text { No. }\end{array}$} & \multicolumn{3}{|c|}{ Fertilizer levels $(\mathrm{Kg} / \mathrm{ha})$} & \multicolumn{3}{|c|}{ Genotypes yield (kg/ha) } \\
\hline & $\mathbf{N}$ & $\mathrm{P} 2 \mathrm{O} 5$ & $\mathrm{~K} 2 \mathrm{O}$ & $\begin{array}{l}\text { 11CM-707 } \\
2015\end{array}$ & $\begin{array}{l}\text { 11CM-707 } \\
2016\end{array}$ & Mean \\
\hline 1 & 0 & 0 & 0 & 580 & 749 & 665 \\
\hline 2 & 0 & 60 & 30 & 600 & 800 & 700 \\
\hline 3 & 20 & 60 & 30 & 710 & 910 & 810 \\
\hline 4 & 40 & 60 & 30 & 570 & 796 & 683 \\
\hline 5 & 20 & 30 & 30 & 610 & 823 & 717 \\
\hline 6 & 20 & 90 & 30 & 620 & 844 & 732 \\
\hline \multirow[t]{3}{*}{7} & 20 & 60 & 60 & 650 & 832 & 741 \\
\hline & \multicolumn{2}{|c|}{ Varieties } & \multicolumn{2}{|c|}{ Fertilizer } & \multicolumn{2}{|l|}{ Interaction } \\
\hline & \multicolumn{2}{|c|}{$\begin{array}{l}\operatorname{LSD}(0.05)=49 \\
\mathrm{CV} \%=4.8\end{array}$} & 26 & & 44 & \\
\hline
\end{tabular}

To optimize the fertilizer requirements, mash genotype 11CM-707 was tested at ten different fertility levels during 2015 and 2016 on sandy loam soil having organic matter $(0.5 \%)$ and available phosphorus $(5.3$ $\left.\mathrm{mg} \mathrm{kg}{ }^{-1}\right)$. On an average of two years study, genotype 11 CM-707 responded better to fertilizer dose of 2060-30 NPK kg ha-1 (Table 9).

\section{Plant protection trials}

Screening trials on mash entries were conducted against diseases at BARI, Chakwal during 2015 and 2016. Genotype 11 CM-707 tested against check cultivars $\mathrm{CH}-\mathrm{Mash}$ and Arooj and was found resistant against yellow mosaic virus, leaf spot and leaf curl virus (Table 10).

Table 10: Screening of mash genotypes against diseases.

$\begin{array}{lllll}\text { S. No. } & \text { Entry } & \text { YMV } & \text { Leaf SPOT } & \text { ULCV } \\ 1 & 10 \mathrm{CM}-707 & 2 & 1 & 1 \\ 2 & 11 \mathrm{CM}-707 & 2 & 2 & 2 \\ 3 & \text { CH-MASH } & 3 & 3 & 2 \\ 4 & \text { Arooj } & 2 & 3 & 2\end{array}$

Note: 1: Immune; 2: Resistant; 3: Tolerant; 4: Susceptible; 5:Highly Susceptible.

Distinctive characteristics of newly developed mash cultivar "Barani Mash"

Mash genotype 11 CM-707 has dark green foliage, semi erect plant type and medium seeded as compared to Arooj. Under rainfed conditions, flowering occurs in 49 days with maturity reached in 78 to 80 days. It has medium sized pods and its 100-seed weight is $4.73 \mathrm{~g}$. This genotype has an average yield of 1743 $\mathrm{kg} \mathrm{ha}{ }^{-1}$ (Table 12). Genotype $11 \mathrm{CM}-707$ has also been proved to a disease and lodging resistant mash genotype. 
Table 11: Average yield of 11-CM-707 in19 experiments.

\begin{tabular}{|c|c|c|c|c|c|}
\hline Year & $\begin{array}{l}\text { Name of } \\
\text { trial }\end{array}$ & $\begin{array}{l}\text { No. of } \\
\text { trial }\end{array}$ & $\begin{array}{l}\text { 11-CM- } \\
707\end{array}$ & $\begin{array}{l}\text { Chakwal } \\
\text { mash }\end{array}$ & Arooj \\
\hline 2012 & PYT & 1 & 628 & 606 & 500 \\
\hline 2013 & RYT & 1 & 1743 & 1577 & 1506 \\
\hline 2014 & MYT & 2 & 528 & 431 & 424 \\
\hline 2015 & NUYT & 5 & 602 & - & 518 \\
\hline 2016 & NUYT & 5 & 1035 & - & 829 \\
\hline 2017 & $\begin{array}{l}\text { Multi loca- } \\
\text { tion trial }\end{array}$ & 3 & 345 & 235 & 238 \\
\hline 2015 & SDT & 1 & 990.5 & - & - \\
\hline 2016 & FT & 1 & 710 & - & - \\
\hline MEAN & & 19 & 823 & 712 & 670 \\
\hline \multicolumn{4}{|c|}{ Yield increase (\%) over Checks } & $+16 \%$ & $+23 \%$ \\
\hline
\end{tabular}

Table 12: Description of mash genotype 11CM-707.

\begin{tabular}{|c|c|c|c|}
\hline $\begin{array}{l}\text { Days to } 50 \% \\
\text { flowering: }\end{array}$ & 49 & Pod size & Medium \\
\hline Growth habit & $\begin{array}{l}\text { Semi } \\
\text { Errect }\end{array}$ & $\begin{array}{l}\text { Seeds per } \\
\text { pod }\end{array}$ & 6.5 \\
\hline Plant height & $46 \mathrm{~cm}$ & Pods per plant & 42 \\
\hline Plant color & $\begin{array}{l}\text { Dark } \\
\text { green }\end{array}$ & $\begin{array}{l}100 \text { seed } \\
\text { weight }\end{array}$ & 4.73 \\
\hline Canopy spread & Medium & $\begin{array}{l}\text { Yield potential } \\
\mathrm{kg} / \mathrm{ha}\end{array}$ & 1743 \\
\hline $\begin{array}{l}\text { Primary branches } \\
\text { attitude }\end{array}$ & $\begin{array}{l}\text { Semi } \\
\text { Errect }\end{array}$ & Lodging & Resistant \\
\hline $\begin{array}{l}\text { No. of pri. branch- } \\
\text { es/plant }\end{array}$ & 2.5 & $\begin{array}{l}\text { Resistant to } \\
\text { diseases }\end{array}$ & $\begin{array}{l}\text { YMV, Leaf spot, } \\
\text { ULCV }\end{array}$ \\
\hline Days to Maturity & $78-80$ & & \\
\hline
\end{tabular}

\section{Novelty Statement}

This paper describes the development of a new high yielding and disease resistant mashbean variety "BARANI MASH" for rainfed areas of Punjab.

\section{Author's Contribution}

GR and JI designed and performed the research. UJ statistically analyzed the data and drafted the manuscript. RM assisted in drafting the manuscript. GS statistically analyzed the data. $\mathrm{FH}$ assisted in designing and performing the research. All authors read and approved the final manuscript. All authors declare that there is no conflict of interests regarding the publication of this article.

\section{Conflict of interest}

The authors have declared no conflict of interest.

\section{References}

AMIS (Agriculture Marketing Information Service). 2019-20. Directorate of Agriculture (Economics and Marketing) Punjab, Lahore, Pakistan.

AMIS (Agriculture Marketing Information Service). 2017-18. Directorate of Agriculture (Economics and Marketing) Punjab, Lahore, Pakistan.

Dasgupta,T.and P.K. Das.1991. Genetic divergence in black gram. Indian J. Agric. Res., 25: 7-13.

Eberhart, S.A. and W.A. Russel. 1966. Stability parameters for comparing varieties. Crop Sci., 6: 36-40. https://doi.org/10.2135/ cropsci1966.0011183X000600010011x

Ghafoor,A.,Z.Ahmad and A. Qayyum.2003.Black gram (Vigna mungo L. Hepper) germplasm catalogue. Plant Genetic Resources Program, PARC/JICA, Islamabad, Pakistan. pp. 75-80.

GOP (Government of Pakistan). 2018-19. Economic survey of Pakistan. Economic Advisory Wing, Finance Division, Islamabad.

Naeem-ud-Din, A. Mahmood, G.S.S. Khattak, I. Saeed and M.F. Hassan. 2009. High yielding groundnut variety Golden. Pak. J. Bot., 41(5): 2217-2222.

Nazir, M.S., 1994. Crop Production, National Book Foundation, Islamabad. pp. 308-313.

Qayyum, A., J. Iqbal, L. Barbanti, A. Sher, G. Shabbir, G. Rabbani, M.K. Rafiq, M.N. Tareen, M.J. Tareen and B.A.Z. Amin. 2019. Mash Bean [Vigna mungo (L.) Hepper] Germplasm evaluation at different ecological conditions of Pakistan. Appl. Ecol. Environ. Res., 17(3): 6643-6654. https://doi.org/10.15666/ aeer/1703_66436654

Shrivastava, S.N., 1977. Effect of row spacing on yield of black gram. Thesis Absts. India, pp. 3-20. 